\title{
Artin Groups of Finite Type with Three Generators
}

\author{
THOMAS BRADY
}

\section{Introduction}

Let $W$ be a finite Coxeter group on three generators $A, B, C$, and consider the set of all possible expressions of the Coxeter element $X=B A C$ in $W$ as a product of three reflections. In Section 3 we will construct a 3 -dimensional CW-complex, $K(W)$, which we can associate to this set in a natural way. (Daan Krammer has informed us that he has also considered this complex and has obtained similar results.) We will show that this complex enjoys two remarkable properties. First, the fundamental group of $K(W)$ is isomorphic to the finite type Artin group determined by $W$; second, $K(W)$ can be given a piecewise Euclidean (PE) metric of nonpositive curvature. Thus, if $G$ is an Artin group of finite type with three generators, then $G$ acts cocompactly on a 3-dimensional PE complex of nonpositive curvature.

The paper is arranged as follows. In Section 2, we make the corresponding construction for two-generator Artin groups and prove that the associated 2-complex has nonpositive curvature. In Section 3, we define the complex $K$ and show that it has the correct fundamental group. In Section 4 , we give $K$ a PE metric and show that it has nonpositive curvature.

We would like to thank the Mathematics Department at Brigham Young University, where most of this work was completed.

\section{Artin Groups of Finite Type with Two Generators}

Let $W$ be a finite Coxeter group on two generators $A$ and $B$; that is, $W=W_{m}$ has a presentation of the form

$$
W_{m}=\left\langle A, B \mid A^{2}=B^{2}=(A B)^{m}=1\right\rangle .
$$

Thus $W_{m}$ is the dihedral group of order $2 m$, which can be thought of as a finite reflection group acting on $\mathbf{R}^{2}$. The elements $A$ and $B$ act as reflections in lines through the origin that make an angle of $\pi / m$ while the element $A B$ acts by rotation through $2 \pi / m$. The corresponding Artin group is the group with the presentation

$$
G_{m}=\langle a, b \mid \operatorname{prod}(a, b ; m)=\operatorname{prod}(b, a ; m)\rangle,
$$

Received September 7, 1999. Revision received March 7, 2000. 
where $\operatorname{prod}(x, y ; k)$ is the word $x y x y x \ldots$ with a total of $k$ letters. There is a canonical surjection from $G_{m}$ to $W_{m}$ taking $a$ to $A$ and $b$ to $B$. We will exhibit a different presentation for $G$ and use it to construct a presentation 2-complex whose universal cover is PE and has nonpositive curvature. The existence of such a 2-complex is not surprising, since the Artin groups are virtually direct products of free groups with $Z$. However, the construction here will motivate the construction for three-generator Artin groups and Proposition 2.1 will be used to prove the equivalence of presentations in Section 3.

Define the abstract group $\Gamma_{m}$ by the presentation

$$
\Gamma_{m}=\left\langle y, a_{1}, a_{2}, \ldots, a_{m} \mid y=a_{1} a_{2}=a_{2} a_{3}=\cdots=a_{m-1} a_{m}=a_{m} a_{1}\right\rangle .
$$

We note that there is also a canonical surjection from $\Gamma_{m}$ to the reflection group $W_{m}$ that takes $a_{1}$ to $A, a_{2}$ to $B, y$ to the rotation $A B$, and the other $a_{i}$ to the other reflections in $W_{m}$. In fact, the following proposition is shown in [4].

Proposition 2.1. For all $m$, the homomorphism $G_{m} \cong \Gamma_{m}$ defined by $a \mapsto a_{1}$ and $b \mapsto a_{2}$ is an isomorphism with inverse defined by

$$
y \mapsto a b, \quad a_{2 k+1} \mapsto(a b)^{-k} a(a b)^{k}, \quad a_{2 k+2} \mapsto(a b)^{-k} b(a b)^{k} .
$$

Next we see that the presentation 2-complex determined by $\Gamma_{m}$ can be given a piecewise Euclidean metric of nonpositive curvature.

TheORem 2.2. The two-generator Artin groups $G_{m}$ act cocompactly by isometries on contractible 2-complexes of nonpositive curvature.

Proof. Let $K_{m}$ be the presentation 2-complex for $\Gamma_{m}$. Thus $K_{m}$ has a single 0 -cell and its 1-cells are labelled $y, a_{1}, \ldots, a_{m}$, with each 1 -cell having both its endpoints attached to the single 0 -cell. For each relation $y=r s$ in the presentation, we attach a 2-cell by gluing the boundary along the path reading $r s y^{-1}$ in the 1-skeleton. We give $K_{m}$ a PE metric by assigning lengths to the 1-cells. Each 1-cell labeled $a_{i}$ is given length 1 and the 1-cell labeled $y$ is given length $\sqrt{2}$, so that each 2-cell becomes a Euclidean triangle with sides 1,1 , and $\sqrt{2}$. We know from [1], for example, that $K_{m}$ will have nonpositive curvature if the vertex link has no embedded loops of length less than $2 \pi$. From Figure 1 we see that this is indeed the case. The universal cover of $K_{m}$ is now the desired 2-complex.

Note. This result can also be deduced from [4], where a slightly different metric is used.

\section{Artin Groups of Finite Type with Three Generators}

Let $W$ be a Coxeter group on three generators $A, B$, and $C$; that is, $W=W_{m, n, p}$ has a presentation of the form

$$
W_{m, n, p}=\left\langle A, B, C \mid A^{2}=B^{2}=C^{2}=(A B)^{m}=(B C)^{n}=(C A)^{p}=1\right\rangle .
$$




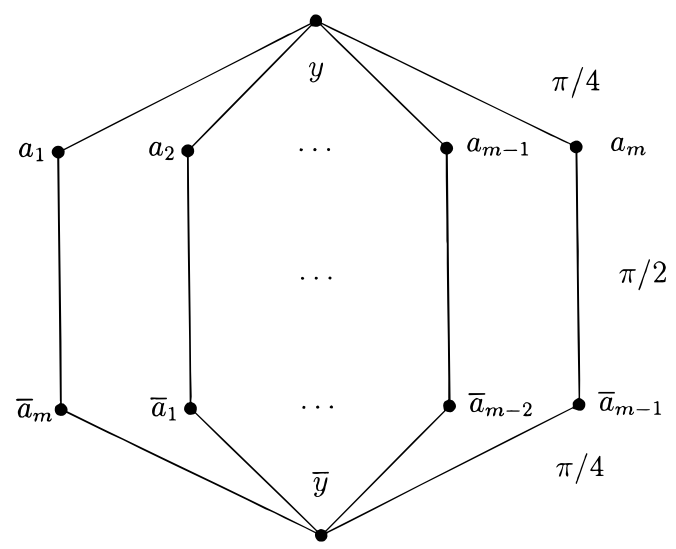

Figure 1

The corresponding Artin group is the group with the presentation

$$
\begin{aligned}
G_{m, n, p}=\langle a, b, c| \operatorname{prod}(a, b ; m) & =\operatorname{prod}(b, a ; m), \\
\operatorname{prod}(b, c ; n) & =\operatorname{prod}(c, b ; n), \\
\operatorname{prod}(c, a ; p) & =\operatorname{prod}(a, c ; p)\rangle .
\end{aligned}
$$

There is a canonical surjection from $G_{m, n, p}$ to $W_{m, n, p}$ taking $a$ to $A, b$ to $B$, and $c$ to $C$. The Artin group $G_{m, n, p}$ is said to have finite type if the corresponding Coxeter group $W_{m, n, p}$ is finite. The groups $W_{m, n, p}$ are finite when the exponents $(m, n, p)$ take on the values $(3,3,2),(3,4,2),(3,5,2)$, and $(2, n, 2)$ for $n \geq 2$.

These Coxeter groups have been much studied, and the following terminology is used. The group $W_{3,3,2}$ is known as the Weyl group $\mathcal{A}_{3}$ or the symmetric group $\Sigma_{4}$. It is the complete symmetry group of the regular tetrahedron. The corresponding Artin group is the braid group on four strands. The group $W_{3,4,2}$ is known as the Weyl group $\mathcal{B}_{3}$. It is the complete symmetry group of the regular cube or octohedron. The group $W_{3,5,2}$ is known as $\mathcal{H}_{3}$ and is the complete symmetry group of the regular dodecahedron or icosohedron. The group $W_{2, n, 2}$ is a direct product of the dihedral group of order $2 n, D_{2 n}=\langle b, c\rangle$, with $\mathbf{Z}_{2}=\langle a\rangle$. Finally, we note that when $(m, n, p)=(2,2,2)$, both the Coxeter group $\left(\mathbf{Z}_{2}\right)^{3}$ and the Artin group $\mathbf{Z}^{3}$ are abelian.

We will use the description of $W$ as a finite reflection group, where the reflections are across planes in $\mathbf{R}^{3}$ through the origin. In Figure 2 we show part of the intersection pattern of the reflection circles on the 2-sphere for the group $W_{3,5,2}$. The 2-sphere is cut open and flattened out onto the plane. The intersection patterns for the other groups can be illustrated with similar diagrams.

Recall that any product of all the standard generators in a finite Coxeter group is called a Coxeter element. These elements were first used by Coxeter in [6]. In $W_{m, n, p}$, any product of the three generators $A, B, C$ is a Coxeter element. All 


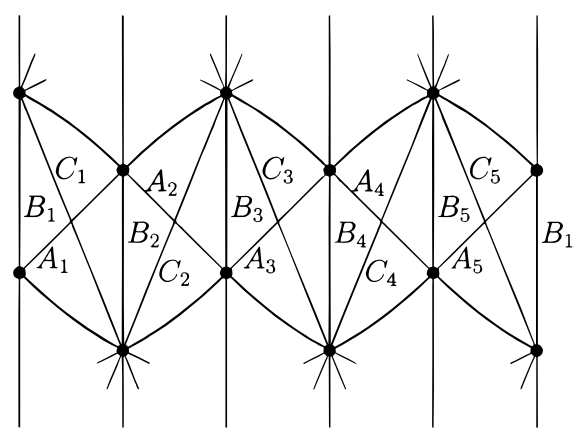

Figure 2

Coxeter elements in $W$ are conjugate in $W$ (see [7, p. 74]), but we will always mean the particular element $X=B A C$.

In order to define the 3-complexes for the three-generator groups, we will use a subset of $W$ that is a natural generalization of the set of generators used to define the groups $\Gamma_{m}$. We call an element $w \in W$ allowable if

(a) $w$ can be expressed as a product of one, two, or three reflections, and

(b) the product in (a) is a prefix of some expression for the element $X$ as a product of three reflections.

We will say that an allowable element has length $k$ if the expression in (a) has $k$ reflections. This notion of length is quite different from the usual length of a word in a Coxeter group. In the symmetric group $\Sigma_{4}$, for example, this length coincides with transposition length for allowable elements. We note that $X$ is the only allowable element of length 3 .

For each $W=W_{m, n, p}$, we define a 3-complex $K=K_{m, n, p}$ that is analogous to the 2-complexes of Section 2. The complex $K$ has a single 0 -cell and a 1-cell for each allowable element of $W$. As before, each 1-cell has both its endpoints attached to the 0 -cell. Observe that $K$ has a 2-cell for each expression of an allowable element of length $k$ as a product of two allowable elements, the sum of whose lengths is $k$. The boundary of the 2-cell consists of three 1-cells, and this gives the prescription for attaching the 2-cell to the 1-skeleton of $K$. Finally, $K$ has a 3-cell for each expression of $X$ as a product of three reflections. If the expression is $X=R S T$ and we define the allowable elements $Y=R S$ and $Z=S T$, then the boundary of the 3-cell consists of the four 2-cells with boundary labels $Y=R S$, $Z=S T, X=Y T$, and $X=R Z$. This specifies the attaching map for the 3-cell. For a specific triple $(m, n, p)$, the cell structure of $K$ can be deduced from one of Tables 1-5, which record all possible expressions for the element $X$ as a product of three reflections. These tables were compiled using induction combined with the following proposition.

Proposition 3.1. Each reflection is allowable. Moreover, every reflection is conjugate to one of $A, B$, or $C$ by some power of the Coxeter element. 
Table $1 G_{3,3,2}$

Reflections: $A_{1}, A_{2}, A_{3}, A_{4}, B_{1}, B_{2}$

Expressions for Coxeter element:

$B_{1} A_{1} A_{3} \quad A_{1} A_{3} B_{2} \quad A_{3} B_{2} A_{2} \quad B_{2} A_{2} A_{4} \quad A_{2} A_{4} B_{1} \quad A_{4} B_{1} A_{3} \quad B_{1} A_{3} A_{1}$ $A_{3} A_{1} B_{2} \quad A_{1} B_{2} A_{4} \quad B_{2} A_{4} A_{2} \quad A_{4} A_{2} B_{1} \quad A_{2} B_{1} A_{1} \quad A_{3} A_{2} A_{1} \quad A_{2} A_{1} A_{4}$ $A_{1} A_{4} A_{3} \quad A_{4} A_{3} A_{2}$

Table $2 G_{3,4,2}$

Reflections: $A_{1}, A_{2}, A_{3}, B_{1}, B_{2}, B_{3}, C_{1}, C_{2}, C_{3}$

Expressions for Coxeter element:

\begin{tabular}{lllllll}
$B_{1} A_{1} C_{1}$ & $A_{1} C_{1} B_{2}$ & $C_{1} B_{2} A_{2}$ & $B_{2} A_{2} C_{2}$ & $A_{2} C_{2} B_{3}$ & $C_{2} B_{3} A_{3}$ & $B_{3} A_{3} C_{3}$ \\
$A_{3} C_{3} B_{1}$ & $C_{3} B_{1} A_{1}$ & $B_{1} C_{1} A_{1}$ & $C_{1} A_{1} B_{2}$ & $A_{1} B_{2} C_{2}$ & $B_{2} C_{2} A_{2}$ & $C_{2} A_{2} B_{3}$ \\
$A_{2} B_{3} C_{3}$ & $B_{3} C_{3} A_{3}$ & $C_{3} A_{3} B_{1}$ & $A_{3} B_{1} C_{1}$ & $C_{1} A_{2} A_{1}$ & $A_{2} A_{1} C_{2}$ & $A_{1} C_{2} A_{3}$ \\
$C_{2} A_{3} A_{2}$ & $A_{3} A_{2} C_{3}$ & $A_{2} C_{3} A_{1}$ & $C_{3} A_{1} A_{3}$ & $A_{1} A_{3} C_{1}$ & $A_{3} C_{1} A_{2}$ & \\
\hline
\end{tabular}

Table $3 G_{3,5,2}$

Reflections: $A_{1}, \ldots, A_{5}, B_{1}, \ldots, B_{5}, C_{1}, \ldots, C_{5}$

Expressions for Coxeter element:

\begin{tabular}{lllllll}
$B_{1} A_{1} C_{1}$ & $A_{1} C_{1} B_{2}$ & $C_{1} B_{2} A_{2}$ & $B_{2} A_{2} C_{2}$ & $A_{2} C_{2} B_{3}$ & $C_{2} B_{3} A_{3}$ & $B_{3} A_{3} C_{3}$ \\
$A_{3} C_{3} B_{4}$ & $C_{3} B_{4} A_{4}$ & $B_{4} A_{4} C_{4}$ & $A_{4} C_{4} B_{5}$ & $C_{4} B_{5} A_{5}$ & $B_{5} A_{5} C_{5}$ & $A_{5} C_{5} B_{1}$ \\
$C_{5} B_{1} A_{1}$ & $B_{1} C_{1} A_{1}$ & $C_{1} A_{1} B_{2}$ & $A_{1} B_{2} C_{2}$ & $B_{2} C_{2} A_{2}$ & $C_{2} A_{2} B_{3}$ & $A_{2} B_{3} C_{3}$ \\
$B_{3} C_{3} A_{3}$ & $C_{3} A_{3} B_{4}$ & $A_{3} B_{4} C_{4}$ & $B_{4} C_{4} A_{4}$ & $C_{4} A_{4} B_{5}$ & $A_{4} B_{5} C_{5}$ & $B_{5} C_{5} A_{5}$ \\
$C_{5} A_{5} B_{1}$ & $A_{5} B_{1} C_{1}$ & $C_{1} A_{2} A_{1}$ & $A_{2} A_{1} C_{2}$ & $A_{1} C_{2} A_{3}$ & $C_{2} A_{3} A_{2}$ & $A_{3} A_{2} C_{3}$ \\
$A_{2} C_{3} A_{4}$ & $C_{3} A_{4} A_{3}$ & $A_{4} A_{3} C_{4}$ & $A_{3} C_{4} A_{5}$ & $C_{4} A_{5} A_{4}$ & $A_{5} A_{4} C_{5}$ & $A_{4} C_{5} A_{1}$ \\
$C_{5} A_{1} A_{5}$ & $A_{1} A_{5} C_{1}$ & $A_{5} C_{1} A_{2}$ & $A_{2} A_{4} A_{1}$ & $A_{4} A_{1} A_{3}$ & $A_{1} A_{3} A_{5}$ & $A_{3} A_{5} A_{2}$ \\
$A_{5} A_{2} A_{4}$ & & & & & & \\
\hline
\end{tabular}

Proof. If $W$ is reducible then this follows easily from the two-generator case, so we will assume that $W$ is irreducible. First note that $B, A$, and $C$ are each allowable, since

$$
X=B A C=A C(C A B A C)=C(C B C)(C A C) .
$$

Next, since $A$ and $C$ commute, our choice of the Coxeter element $X=B A C$ means that $X=X_{1} X_{2}$, where the factors of $X_{1}=B$ are commuting reflections and likewise the factors of $X_{2}=A C$. Coxeter elements of this type were used to 
Table $4 G_{2,2 k, 2}$

\begin{tabular}{|c|c|c|c|c|c|c|}
\hline \multicolumn{7}{|c|}{ Expressions for Coxeter element: } \\
\hline$B_{1} A_{1} C_{1}$ & $A_{1} C_{1} B_{2}$ & $C_{1} B_{2} A_{1}$ & $\ldots$ & $B_{k} A_{1} C_{k}$ & $A_{1} C_{k} B_{1}$ & $C_{k} B_{1} A_{1}$ \\
\hline$B_{1} C_{1} A_{1}$ & $C_{1} A_{1} B_{2}$ & $A_{1} B_{2} C_{2}$ & $\ldots$ & $B_{k} C_{k} A_{1}$ & $C_{k} A_{1} B_{1}$ & $A_{1} B_{1} C_{1}$ \\
\hline
\end{tabular}

Table $5 G_{2,2 k+1,2}$

\begin{tabular}{ccc}
\hline \hline Reflections: $A_{1}, B_{1}, \ldots, B_{2 k+1}$ \\
\hline \multicolumn{3}{c}{ Expressions for Coxeter element: } \\
$B_{1} A_{1} B_{k+2}$ & $A_{1} B_{k+2} B_{2}$ & $B_{k+2} B_{2} A_{1}$ \\
$B_{2} A_{1} B_{k+3}$ & $A_{1} B_{k+3} B_{3}$ & $B_{k+3} B_{3} A_{1}$ \\
$\vdots$ & $\vdots$ & $\vdots$ \\
$B_{2 k+1} A_{1} B_{k+1}$ & $A_{1} B_{k+1} B_{1}$ & $B_{k+1} B_{1} A_{1}$ \\
\hline
\end{tabular}

great advantage by Steinberg in [8]. For such a Coxeter element it follows from $[8$, Cor. 4.6] that the reflections in $W$ are

$$
B, B A B, B A C A B, B A C B C A B, B A C B A B C A B, \ldots,
$$

which can be rewritten as

$$
B, X A X^{-1}, X C X^{-1}, X B X^{-1}, X^{2} A X^{-2}, \ldots
$$

Thus each reflection in $W$ is a conjugate of one of three allowable reflections ( $A, B$, or $C$ ) by some power of the Coxeter element $X$. However, each such element is allowable, since whenever $X=R S T$ we have

$$
X=X^{-k}(X) X^{k}=\left(X^{-k} R X^{k}\right)\left(X^{-k} S X^{k}\right)\left(X^{-k} T X^{k}\right) .
$$

We are now in a position to prove the analog of Proposition 2.1.

Theorem 3.2. If $W_{m, n, p}$ is finite, then $\pi_{1}\left(K_{m, n, p}\right) \cong G_{m, n, p}$.

Proof. Computing $\pi_{1}\left(K_{m, n, p}\right)$ from the 2-skeleton and eliminating the generators corresponding to length-2 allowable elements, we find that $\pi_{1}\left(K_{m, n, p}\right)$ has the following presentation. There is one generator $r$ for each reflection $R$ in $W$ and an extra generator $x$ corresponding to the Coxeter element $X=B A C$ in $W$. The relations of $\pi_{1}\left(K_{m, n, p}\right)$ are all of the form $x=r s t$, where $r, s, t$ are generators that correspond to allowable elements of length 1 for which the corresponding reflections in $W$ satisfy $X=R S T$. 
In each of the Artin groups $G$ under consideration, we define $x=b a c$ and $r_{i}=$ $x^{1-i} r x^{i-1}$ for $r \in\{a, b, c\}$, so that $a_{1}=a, b_{1}=b$, and $c_{1}=c$. Under the quotient map from $G$ to $W, x$ maps to the Coxeter element and each $r_{i}$ maps to a reflection. We will refer to $x$ as the lift of the Coxeter element and to each of the $r_{i}$ as lifts of reflections.

Our first step will be to establish a set of identities in $G$ of the form $x=r s t$, where $r, s, t$ are lifts of reflections. Since $W$ is a homomorphic image of $G$, each such identity will give a factoring of the Coxeter element as a product of three reflections. We will see that our list of identities exhausts the set of possible factorings of the Coxeter element and thus we will have a well-defined surjective homomorphism from $\pi_{1}\left(K_{m, n, p}\right)$ to $G$.

First observe that, whenever $x=r s t$ and $x=s t u$,

$$
r x=r(s t u)=(r s t) u=x u
$$

so that $u=x^{-1} r x$. Thus we can write

$$
x=b_{i} a_{i} c_{i}=a_{i} c_{i} b_{i+1}=c_{i} b_{i+1} a_{i+1} .
$$

Using that $a c=c a$ in each Artin group, we deduce

$$
x=b_{i} c_{i} a_{i}=c_{i} a_{i} b_{i+1}=a_{i} b_{i+1} c_{i+1} .
$$

Equating expressions for $x$ (starting with $c_{i}$ ), we obtain

$$
a_{i} b_{i+1}=b_{i+1} a_{i+1} .
$$

We now look at each specific group in turn.

Case 1: $G$ is irreducible. Since the group generated by $b_{i+1}$ and $a_{i+1}$ is a twogenerator Artin group of type $G_{3}$, we deduce from equation (4) and Proposition 2.1 that

$$
a_{i} b_{i+1}=b_{i+1} a_{i+1}=a_{i+1} a_{i} .
$$

This yields more expressions for $x$ :

$$
x=c_{i} a_{i+1} a_{i}=a_{i+1} a_{i} c_{i+1}=a_{i} c_{i+1} a_{i+2} .
$$

Equating expressions for $x$ starting with $a_{i}$, we have

$$
a_{i-1} c_{i}=c_{i} b_{i+1}=b_{i+1} c_{i+1}=c_{i+1} a_{i+2} .
$$

Case 1(a): $G=G_{3,5,2}$. Here the group generated by $b_{i+1}$ and $c_{i+1}$ is a twogenerator Artin group of type $G_{5}$, so we deduce from Proposition 2.1 and equation (6) that

$$
a_{i-1} c_{i}=c_{i} b_{i+1}=b_{i+1} c_{i+1}=c_{i+1} a_{i+2}=a_{i+2} a_{i-1} .
$$

This gives more expressions for $x$ :

$$
x=a_{i} a_{i+2} a_{i-1}=a_{i+2} a_{i-1} a_{i+1}=a_{i-1} a_{i+1} a_{i+3} .
$$

Equating expressions for $x$, starting with $a_{i}$ once again, we obtain 


$$
a_{i+2} a_{i-1}=a_{i-3} a_{i-1}=a_{i+2} a_{i+4},
$$

from which we conclude $a_{i}=a_{i+5}$. From this and equation (5) it follows that $c_{i}=c_{i+5}$, and from this and equation (2) we get $b_{i}=b_{i+5}$. Thus, $x^{5}$ is central; together with equations (2), (3), (5), and (7), this gives the expressions for $x$ corresponding to Table 3 .

Case 1(b): $G=G_{3,4,2}$. Now the group generated by $b_{i+1}$ and $c_{i+1}$ is a twogenerator Artin group of type $G_{4}$, so we deduce from Proposition 2.1 and equation (6) that $a_{i-1}=a_{i+2}$ or $a_{i}=a_{i+3}$. Together with equation (5), this yields $c_{i}=$ $c_{i+3}$ and from equation (2) we have $b_{i}=b_{i+3}$. Thus $x^{3}$ is central, and together with equations (2), (3), and (5) this gives the expressions for $x$ corresponding to Table 2 .

Case 1(c): $G=G_{3,3,2}$. Now the group generated by $b_{i+1}$ and $c_{i+1}$ is a twogenerator Artin group of type $G_{3}$, so we deduce from Proposition 2.1 and equation (6) that $c_{i}=a_{i+2}$ and $c_{i+1}=a_{i-1}$. From this we conclude that $a_{i}=a_{i+4}$. Finally, equation (3) gives

$$
x=b_{i+2} c_{i+2} a_{i+2}=b_{i+2} a_{i+4} c_{i}=b_{i+2} a_{i} c_{i} ;
$$

together with equation (2), this gives $b_{i+2}=b_{i}$. Thus $x^{4}$ is central, and together with equations (2), (3), and (5) this gives the expressions for $x$ corresponding to Table 1.

Case 2: $G$ is reducible. Here the group generated by $b_{i+1}$ and $a_{i+1}$ is a twogenerator Artin group of type $G_{2}$. Thus we deduce from Proposition 2.1 and equation (4) that $a_{i+1}=a_{i}$. Equating expressions for $x$ starting with $a_{i}$ yields

$$
c_{i} b_{i+1}=b_{i+1} c_{i+1}\left(=c_{i+1} b_{i+2}=b_{i+2} c_{i+2}\right) .
$$

However, the group generated by $b_{i+1}$ and $c_{i+1}$ is a two-generator Artin group of type $G_{n}$.

Case 2(a): $n$ is even. If $n=2 k$ then $b_{i+k}=b_{i}, c_{i+k}=c_{i}$, and $x^{k}$ is central. Together with equations (2) and (3), this gives the expressions for $x$ corresponding to Table 4.

Case 2(b): $n$ is odd. If $n=2 k+1$ then $c_{i}=b_{i+k+1}, b_{i+n}=b_{i}$, and $x^{n}$ is central. Together with equation (2), this gives the expressions for $x$ corresponding to Table 5 .

These expressions correspond to all possible factorings of the Coxeter element $X$ in $W$. For a given $W$ and a given reflection $R$, we find at least one factoring with first factor $R$ by Proposition 3.1. If $W=R S T$ then all the possible factorings starting with $R$ correspond to the number of ways of factoring the rotation $S T$ in the dihedral group generated by $S$ and $T$.

Hence we get a well-defined surjective homomorphism from $\pi_{1}\left(K_{m, n, p}\right)$ to $G$ given by

$$
a_{i} \rightarrow x^{1-i} a x^{i-1}, \quad b_{i} \rightarrow x^{1-i} b x^{i-1}, \quad c_{i} \rightarrow x^{1-i} c x^{i-1},
$$


where $x=b a c$. The inverse homomorphism that is given by $a \rightarrow a_{1}, b \rightarrow b_{1}$, and $c \rightarrow c_{1}$ is well-defined by Proposition 2.1 and is surjective by the proof of Proposition 3.1.

\section{The Piecewise Euclidean Metric on $K$}

Since each 3-cell of $K$ is combinatorially a 3-simplex, we can give $K$ the structure of a PE cell complex by specifying the lengths of 1-cells. Each 1-cell corresponds to a generator that is an allowable element of $W$ of a given length $k$, where $k=$ 1,2 , or 3 . We give the corresponding 1-cell the length $\sqrt{k}$. Thus each 3 -cell in $K$ can be given the metric of the Euclidean simplex on the vertices $(0,0,0),(1,0,0)$, $(1,1,0)$, and $(1,1,1)$ in $\mathbf{R}^{3}$, where the 1-cells of length 1 correspond to reflections, the 1-cells of length $\sqrt{2}$ correspond to allowable elements of length 2 , and the 1-cell of length $\sqrt{3}$ corresponds to $x$. We note that this Euclidean tetrahedron is part of a standard subdivision of the unit cube into six congruent tetrahedra.

ExAmPLE. We note that, for the group $G_{2,2,2}$, there are precisely six 3-cells in $K$ corresponding to the six expressions for $x$ in $W_{2,2,2}$ as $b a c, b c a, c b a, c a b, a c b$, or $a b c$. These six 3-cells fit together to give a subdivision of the usual flat 3-torus with fundamental group $G_{2,2,2}=Z^{3}$.

EXAMPLE. In general, the complex $K_{n, 2,2}$, is isometric to a product of the metric 2-complex $K_{n}$ described in Theorem 2.2 with a circle of length 1 . The isometry $K_{n} \times S^{1} \rightarrow K_{n, 2,2}$ can be described at the level of 3-cells by subdividing the product of a 2-cell with a 1-cell into three 3-cells-in the standard way that a triangular prism is subdivided simplicially. Specifically, if the 2-cell from $K_{n}$ is labeled by $Y=R S(=B A)$ and if the 1-cell is labeled by the central element $C$, then the corresponding 3-cell in $K_{n} \times S^{1}$ is mapped to the union of the 3-cells labeled by $X=R S C, X=R C S$, and $X=C R S$.

Next we establish two lemmas regarding the local geometry in $K$. (See [5] for definition and properties of spherical joins.) Let $v$ be the single vertex in $K$, and let $S$ be its star and $L$ its link. Let $x^{*}$ be the vertex in $L$ corresponding to the edge $x$ in $K$, let $\Sigma$ be the star of the vertex $x^{*}$ in $L$, and let $\Lambda$ be the link of $x^{*}$ in $L$. Thus each 1-cell in $\Lambda$ joins a vertex labeled $R_{i}$ to a vertex labeled $R_{i} R_{j}$, where $R_{i}$ and $R_{j}$ are reflections and $R_{i} R_{j}$ is allowable of length 2 . The 1-complex $\Lambda$ is shown in Figure 3 for the group $G_{3,5,2}$, where the allowable elements of length 2 are

$$
\begin{aligned}
Y_{i} & =B_{i} A_{i}=A_{i} A_{i-1}=A_{i-1} B_{i}, \\
Z_{i} & =B_{i} C_{i}=C_{i} A_{i+1}=A_{i+1} A_{i-2}=A_{i-2} C_{i-1}=C_{i-1} B_{i}, \\
W_{i} & =A_{i} C_{i}=C_{i} A_{i} .
\end{aligned}
$$

We note that the 1-complex for $G_{3,3,2}$ is precisely the link that occurs in [2].

LEMмA 4.1. There is an isometry between the link $L$ and the spherical join of a copy of the 0-sphere with the 1-complex $\Lambda$. 


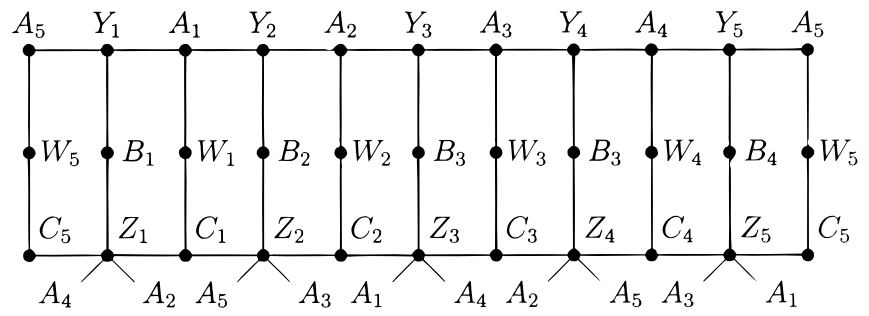

Figure 3

Proof. It follows from equation (1) that the face with label $Z=S T$ of the 3-cell corresponding to the expression $X=R S T$ is incident on exactly one other 3-cell, namely the one corresponding to $X=S T U$, where $U=X^{-1} R X$. Similarily, the face labeled $Y=R S$ is incident on the single other 3-cell $X=Q R S$, where $Q=X T X^{-1}$. Thus the universal cover $\tilde{K}$ of $K(G)$ is a union of infinite triangular prisms. It is this structure that will give us the required isometry.

Let $\tilde{v}$ be a fixed vertex in $\tilde{K}$. We will study the star of $\tilde{v}$ in $\tilde{K}$ since it is isometric to the star of $v$ in $K$. Every 1-cell of $\Lambda$ joins a vertex corresponding to a reflection $R$ to a vertex corresponding to an allowable element $R S$. Since $R S$ is allowable, there is a reflection $T$ satisfying $X=R S T$. Let $U=X T X^{-1}, V=X S X^{-1}$, and $W=X R X^{-1}$ so that

$$
X=W V U=V U R=U R S=R S T .
$$

We consider the seven vertices in $\tilde{K}$ given by

$$
X^{-1} \tilde{v}, \quad(V U)^{-1} \tilde{v}, \quad U^{-1} \tilde{v}, \quad \tilde{v}, \quad R \tilde{v}, \quad(R S) \tilde{v}, \quad X \tilde{v} .
$$

The part of the star of $\tilde{v}$ in $\tilde{K}$ consisting of those simplices whose vertices belong to this set of seven is a union of four 3-simplices corresponding to the four expressions for $X$ in equation (8). Furthermore, this part of the star is isometric to the piece of $\mathbf{R}^{3}$ shown in Figure 4, where the seven vertices are mapped to $(-1,-1,-1),(0,-1,-1),(0,0,-1),(0,0,0),(1,0,0),(1,1,0)$, and $(1,1,1)$, respectively. Hence, this part of the star of $\tilde{v}$ is isometric to the set of directions at $(0,0,0)$ pointing into the region defined by the inequalities $y+z \leq 0$ and $x+y \leq$ 0 . Both of the bounding planes of this region contain the vertices $(-1,-1,-1)$, $(0,0,0)$, and $(1,1,1)$. The plane $y+z=0$ also contains the vertices $(0,-1,-1)$ and $(1,0,0)$, while the plane $x+y=0$ contains $(0,0,-1)$ and $(1,1,0)$. This part of the link thus contributes a segment of a 2 -sphere that is the orthogonal suspension of a copy of the 0 -sphere with a segment of a circle of length $\pi / 3$. The 0 -sphere corresponds to the two points $(-1,-1,-1)$ and $(1,1,1)$, while the segment lies in the plane $x+y+z=0$ between the planes $x+y=0$ and $y+z=$ 0 , which make an angle of $\pi / 3$ between them.

Because (a) every 1-cell of $\Lambda$ contributes precisely one such segment to the star of $\tilde{v}$ and (b) each 3-cell in the star of $\tilde{v}$ lies in exactly one such segment, the 


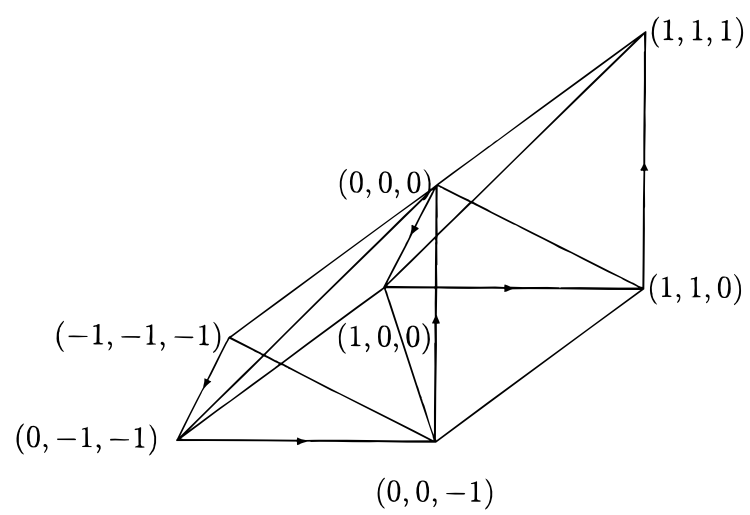

Figure 4

correspondence induces an isometry from $L$ to the spherical join of $S^{0}$ with $\Lambda$ if each 1-cell of $\Lambda$ is given length $\pi / 3$.

LEMMA 4.2. For each 1-complex $\Lambda$, there are no embedded loops with less than six 1-cells.

Proof. The 1-complex $\Lambda$ is the geometric realization of the poset whose elements are the allowable elements of $W$ of length 1 or 2 , with the partial order $P<Y$ if and only if $P$ is allowable of length $1, Y$ is allowable of length 2 , and $Y=P Q$ for some allowable element $Q$ of length 1 . It follows that each circuit in $\Lambda$ traverses an even number of 1-cells and that there are no embedded circuits of length 2 . Hence it suffices to show that there are no embedded circuits of length 4. Suppose such a circuit exists. Then there are allowable elements $P$ and $Q$ of length 1 and allowable elements $Y$ and $Z$ of length 2 with $P<Y, P<Z, Q<Y$, and $Q<Z$. The elements $P$ and $Q$ correspond to reflection planes in $\mathbf{R}^{3}$ through the origin, while $Y$ and $Z$ correspond to lines in $\mathbf{R}^{3}$ where reflection planes intersect. The relations $P<Y$ and $Q<Y$ mean that the planes corresponding to $P$ and $Q$ intersect along the line corresponding to $Y$; similarily, these planes also intersect along the line corresponding to $Z$. Thus these two lines coincide. This gives a contradiction, since there is at most one allowable element of length 2 with fixed set a given line in $\mathbf{R}^{3}$.

THEOREM 4.3. The 3-generator Artin groups $G_{m, n, p}$ of finite type act cocompactly by isometries on contractible 3-complexes of nonpositive curvature.

Proof. The desired 3-complex will be the universal cover of $K_{m, n, p}$. We recall from [1] that a PE cell complex will be CAT(0) if the links of vertices are CAT(1). Lemma 4.1 exhibits this link as a spherical join of a copy of $S^{0}$ with a 1-complex $\Lambda$. By [5], $L$ will be CAT(1) provided that $\Lambda$ is a CAT(1) 1-complex; by [1], $\Lambda$ will be CAT(1) provided $\Lambda$ contains no embedded loops of length less than $2 \pi$. 
Since each edge has length $\pi / 3$, this is equivalent to there being no embedded loops with fewer than six 1-cells. This is established in Lemma 4.2.

REMARK. It is possible to extend the definition of $K(W)$ and its PE structure to finite Coxeter groups $W$ of higher rank. However, the problem of deciding whether or not $K(W)$ has nonpositive curvature is much more difficult in these cases. If $W$ has rank $n$, then the analog of the complex we here called $\Lambda$ is a finite piecewise spherical complex of rank $n-2$. If this complex is CAT(1) then $K(W)$ is $\operatorname{CAT}(0)$. However, it is difficult in general to show that a given PS complex is CAT(1), and few general results of this type exist. We have used one of the simplest-namely, that when the dimension is 1 it suffices to check that there are no embedded short loops. Nonetheless, it can be shown (see [3]) that $K(W)$ is contractible in the braid group case, where $W$ is a symmetric group of any rank.

\section{References}

[1] W. Ballmann, Singular spaces of nonpositive curvature, Progr. Math., 83, Birkhäuser, Boston, 1990.

[2] T. Brady, Automatic structures on Aut $\left(F_{2}\right)$, Arch. Math. (Basel) 63 (1994), 97102.

[3] - A partial order on the symmetric group and new $K(\pi, 1)$ 's for the braid groups, preprint, 1999.

[4] T. Brady and Jon McCammond, Three-generator Artin groups of large type are biautomatic, J. Pure Appl. Algebra 151 (2000), 1-9.

[5] R. Charney and M. W. Davis, Singular metrics of nonpositive curvature on branched covers of Riemannian manifolds, Amer. J. Math. 115 (1993) 929-1009.

[6] H. S. M. Coxeter, Discrete groups generated by reflections, Ann. Math. 35 (1934), 588-621.

[7] J. E. Humphries, Reflection groups and Coxeter groups, Cambridge Stud. Adv. Math., 29, Cambridge Univ. Press, Cambridge, U.K., 1990.

[8] R. Steinberg, Finite reflection groups, Trans. Amer. Math. Soc. 91 (1959), 493-504.

School of Mathematical Sciences

Dublin City University

Glasnevin

Dublin 9

Ireland

tom.brady@dcu.ie 